\title{
Composição química e aceitabilidade de biscoitos elaborados com farinha de banana verde
}

\section{Chemical composition and acceptability of cookies produced with green banana flour}

\author{
Juliana dos Santos Vilar ${ }^{1 *}$, Rodrigo Santos Aranha ${ }^{2}$, Deise Kelly de Oliveira Cardoso ${ }^{2}$, Rafael \\ Bizarelo Ribeiro dos Santos ${ }^{2}$, Omara Machado Araujo de Oliveira ${ }^{2}$
}

\begin{abstract}
RESUMO
O objetivo deste trabalho foi elaborar biscoitos isentos de glúten com farinha de banana verde (FBV), além de avaliar sua composição química e aceitabilidade. Foram elaboradas três formulações: formulação padrão (FP), formulação experimental 1 (F1) e formulação experimental 2 (F2). Na F1, a farinha de trigo foi substituída por $75 \%$ de FBV e $25 \%$ de farinha de arroz, enquanto na F2 essa substituição foi total pela FBV. Foram realizadas análises de composição físico-química e sensorial dos biscoitos e os resultados avaliados utilizando o programa estatístico GraphPad Prism6®. A composição centesimal mostrou que os biscoitos apresentaram baixo teor de umidade e cinzas, e considerável teor de carboidratos e lipídios. Em relação à análise sensorial, participaram do estudo 134 provadores não treinados, sendo a maioria do sexo feminino (78\%), com idade média de 18 a 25 anos (80\%), universitários (74\%), com renda média de 1 a 5 salários mínimos por mês (67\%). Todos os participantes relataram o hábito de consumir biscoitos. O índice de aceitabilidade para os diferentes atributos (cor, sabor, aroma e textura) foi acima de $70 \%$, sendo considerado, portanto, satisfatório. Os biscoitos com FBV proporcionaram uma alternativa de produto isento de glúten.
\end{abstract}

Palavras-chave: Nutrição; Celíaco; Alimentos funcionais.

\section{ABSTRACT}

The aim of this work was to produce and evaluate the acceptability of gluten-free cookies enriched with green banana flour (FBV), in addition to evaluate their chemical composition and acceptability. Three formulations were prepared: standard formulation (FP), experimental formulation 1 (F1) and experimental formulation 2 (F2). In F1, wheat flour was replaced by $75 \%$ of FBV and $25 \%$ of rice flour, while in F2 this replacement was total by FBV. Physicochemical composition and sensory analysis were assessed and the results evaluated using the software GraphPad Prism6®. The centesimal composition showed that the cookies had low moisture and ash contents, and considerable carbohydrate and lipid content. Regarding sensory analysis, 134 untrained tasters participated in the study, most of them female (78\%), with an average age of 18 to 25 years (80\%), university students (74\%), with an average income of 1 to 5 minimum wages per month $(67 \%)$. All participants reported the habit of consuming cookies. The acceptability index for the different attributes (color, flavor, aroma and texture) was above $70 \%$, being considered satisfactory. The cookies prepared with FBV provided an alternative gluten-free product.

Keywords: Nutrition; Celiac; Functional foods.

\footnotetext{
${ }^{1}$ Universidade Federal do Rio de Janeiro (UFRJ), Rio de Janeiro, RJ.

*E-mail: julianavilar@nutricao.ufrj.br

${ }^{2}$ Centro Universitário IBMR, Rio de Janeiro, RJ.
} 


\section{INTRODUÇÃO}

A doença celíaca (DC) é uma doença inflamatória desencadeada pela exposição ao glúten em indivíduos com susceptibilidade genética (AL-BAWARDY et al., 2017). $\mathrm{Na}$ DC ocorre uma atrofia parcial ou total das vilosidades intestinais que, consequentemente, irá prejudicar a absorção digestiva (RODRIGUES, 2013).

O glúten pode ser encontrado em alimentos como trigo, centeio, cevada e aveia, que ao serem ingeridos por celíacos podem gerar sintomas como diarreia, perda de peso, dor abdominal e má absorção de nutrientes. Entretanto, os celíacos também podem apresentar sinais ou sintomas não específicos da doença, como anemia ferropriva isoladamente (AL-BAWARDY et al., 2017).

A prevalência da DC é de $0,3 \%$ a $1,0 \%$ da população europeia ou de ancestralidade europeia, podendo ter casos sem diagnóstico. No Brasil ainda não há dados oficiais de quantos brasileiros sofrem deste problema, porém estima-se que 300 mil brasileiros são portadores da doença, com maior prevalência na região sudeste e mais frequentes em mulheres e pessoas de cor branca (ARAÚJO et al., 2010).

O único tratamento para a doença celíaca é a exclusão dos alimentos fontes de glúten. No tratamento da DC os pacientes têm que escolher os seus alimentos de forma adequada para que não sofram as consequências da exposição a esta proteína. Devido à grande quantidade de alimentos fontes de glúten, especialmente nos processados, há uma dificuldade para seguir uma dieta isenta de glúten (AL-BAWARDY et al., 2017).

No entanto, o valor nutricional dos produtos alimentícios isentos de glúten costuma ser uma limitação enfrentada pelos celíacos, pois esses produtos geralmente são desenvolvidos a partir de farinhas refinadas e amidos, que não são enriquecidos ou fortificados e, portanto, não possuem a mesma quantidade de nutrientes disponíveis em alimentos correspondentes que contêm glúten (GALLAGHER et al., 2004).

Dentre as matérias-primas viáveis para a elaboração de produtos sem glúten, a farinha de banana verde pode ser obtida por secagem natural ou artificial, de frutos de banana. Essa farinha é obtida a partir da desidratação da polpa da banana verde e pode substituir o uso de outras farinhas por ter um sabor suave e, com isso, não alterar esta característica sensorial do alimento (ANDRADE, 2013).

O constituinte principal da banana verde é o amido resistente. Durante o processo digestivo o amido resistente não é digerido e, desta forma, contribui com ações benéficas para o organismo, como: efeitos sobre o índice glicêmico e resposta insulínica, prevenção 
e desenvolvimento de diabetes tipo 2, acúmulo de gordura corporal, redução do colesterol sanguíneo, melhoria do trânsito intestinal, redução do risco de ocorrência do câncer de cólon intestinal, entre outros (RANIERI; DELANI, 2014; PERUCHA, 2005).

Neste contexto, buscar alternativas de produtos isentos de glúten e, tendo em vista a quantidade de celíacos na população brasileira, esse estudo tem como objetivo elaborar e avaliar a composição centesimal e sensorial de biscoitos elaborados com FBV.

\section{MATERIAL E MÉTODOS}

\section{Elaboração dos biscoitos}

Foram realizados estudos preliminares para testar receitas de biscoitos do tipo amanteigado, sendo selecionada uma receita para utilizar como formulação padrão (FP), sendo elaboradas três formulações de biscoitos: formulação padrão (FP), formulação experimental $1(\mathrm{~F} 1)$ e formulação experimental $2(\mathrm{~F} 2)$. Na F1, a farinha de trigo foi substituída por $75 \%$ de pela farinha de banana verde (FBV) e $25 \%$ de farinha de arroz, enquanto na F2 essa substituição foi total pela FBV (Tabela 1).

Tabela 1 - Formulação dos biscoitos padrão (FP) e biscoitos experimentais (FE1 e FE2)

\begin{tabular}{cccc}
\hline Ingredientes & $\begin{array}{c}\text { Formulação } \\
\text { padrão (FP) }\end{array}$ & $\begin{array}{c}\text { Formulação } \\
\text { experimental 1 } \\
(\mathrm{F} 1)\end{array}$ & $\begin{array}{c}\text { Formulação } \\
\text { experimental 2 } \\
\text { (F2) }\end{array}$ \\
\hline Amido de milho & 200 & 200 & 200 \\
Farinha de trigo & 65 & 0 & 0 \\
Farinha de banana verde & 0 & 48,75 & 65 \\
Farinha de arroz & 0 & 16,25 & 0 \\
Margarina & 185 & 185 & 185 \\
Coco ralado & 25 & 25 & 25 \\
Açúcar & 80 & 80 & 80 \\
\hline
\end{tabular}

Fonte: Autoria própria.

Os biscoitos padrão e experimental foram produzidos no Laboratório de Ciência de Alimentos do Centro Universitário IBMR - Campus Barra, com auxílio de utensílios e equipamentos deste laboratório que não entraram em contato com ingredientes fonte de glúten. O preparo consistiu na pesagem e reunião de todos os ingredientes em uma batedeira doméstica. Em seguida, a massa foi modelada em biscoitos e assada à temperatura média de $180^{\circ} \mathrm{C}$ entre 15 a 20 minutos. 


\section{Composição centesimal}

A determinação de umidade, cinzas, proteínas, lipídios e fração NIFEXT seguiram os protocolos estabelecidos pelo Instituto Adolph Lutz (2008). A umidade foi determinada por gravimetria, após dessecação em estufa a $105^{\circ} \mathrm{C}$, até peso constante. Já as cinzas foram determinadas com base na perda de peso que ocorre quando a amostra é incinerada a $500-550^{\circ} \mathrm{C}$. A determinação de proteínas será realizada pelo método de Kjeldahl, os lipídios determinados pelo método de Soxhlet. Já os glicídios totais foram determinados pelo cálculo da diferença, uma vez conhecidos os valores das frações precedentes.

\section{Avaliação sensorial}

Os biscoitos foram avaliados sensorialmente por meio do teste de aceitação com uso da escala hedônica estruturada de 9 pontos, ancorada nos termos: desgostei muitíssimo (1) e gostei muitíssimo (9), para os atributos aceitação global, sabor e consistência. A intenção de compra foi avaliada através da escala hedônica estruturada de sete pontos, ancorada nos termos: nunca compraria (1) e sempre compraria (7). Também foi avaliada a intenção de compra através da escala hedônica estruturada de sete pontos, ancorada nos termos: "nunca compraria" e "sempre compraria" (DUTCOSKY, 2013).

Provadores não treinados receberam as amostras de forma monádica, distribuídas em guardanapos descartáveis, codificados com algarismos de três dígitos, acompanhada da ficha de avaliação e um termo de consentimento livre e esclarecido.

\section{Análise dos resultados}

Os resultados das análises de composição centesimal para cada amostra de biscoito (padrão e experimental) foram expressos com o cálculo da média e desvio padrão de três replicatas.

Os resultados da análise sensorial foram avaliados por meio de análises estatísticas descritivas. Para o cálculo do Índice de Aceitabilidade (IA) do produto foi adotada a expressão: IA $(\%)=\mathrm{Ax} 100 / \mathrm{B}$, em que, $\mathrm{A}=$ nota média obtida para o produto e $\mathrm{B}=$ nota máxima dada ao produto. O IA com boa repercussão tem sido considerado $\geq 70 \%$ (DUTCOSKY, 2013). O teste de intenção de compra foi expresso em frequência.

Para a comparação entre os biscoitos foi realizada análise de variância (ANOVA) e teste de Tukey para revelar as medidas estatisticamente diferentes. Foi utilizado o nível 
de significância de 0,05 . Todas as análises estatísticas foram realizadas no software GraphPad Prism6®.

\section{RESULTADOS E DISCUSSÃO}

Os biscoitos elaborados ficaram com aspecto característico esperado para o tipo de produto (Figura 1).

Figura 1 - Aspectos dos biscoitos elaborados.

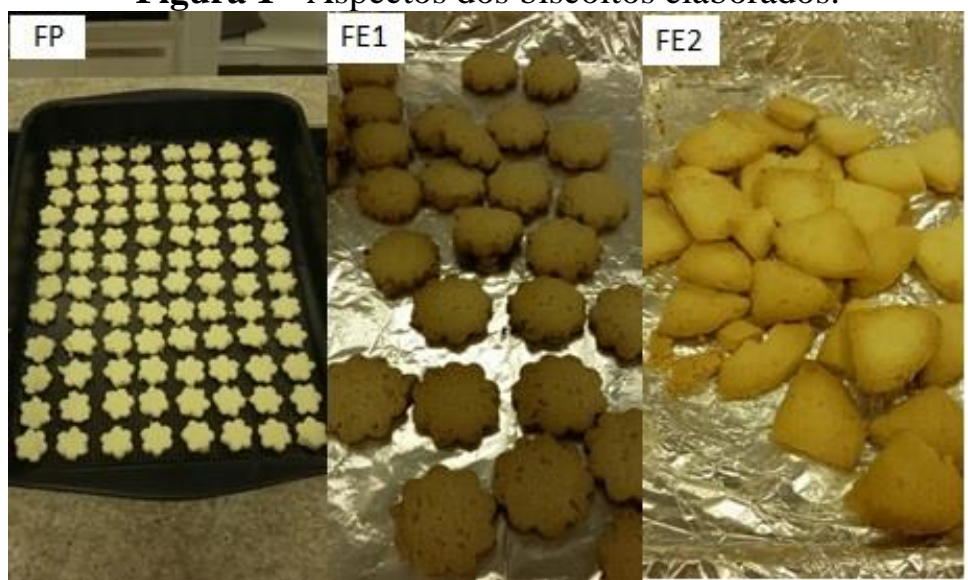

Fonte: Autoria própria.

Na tabela 1, é possível observar a composição nutricional dos biscoitos elaborados no presente trabalho.

Tabela 1 - Composição nutricional dos biscoitos padrão (FP) e biscoitos experimentais (FE1 e

\begin{tabular}{cccc}
\multicolumn{3}{c}{ FE2 $)$} \\
\hline Componentes & FP $(\mathbf{g} / \mathbf{1 0 0 g})$ & FE1 $(\mathbf{g} / \mathbf{1 0 0 g})$ & FE2 $(\mathbf{g} / \mathbf{1 0 0 g})$ \\
& Média \pm DP & Média \pm DP & Média \pm DP \\
\hline Umidade & $3,20 \pm 0,06^{\mathrm{a}}$ & $3,18 \pm 0,08^{\mathrm{a}}$ & $3,20 \pm 0,04^{\mathrm{a}}$ \\
Cinzas & $0,15 \pm 0,09^{\mathrm{a}}$ & $0,57 \pm 0,03^{\mathrm{b}}$ & $0,84 \pm 0,05^{\mathrm{c}}$ \\
Proteínas & $8,10 \pm 0,35^{\mathrm{a}}$ & $5,57 \pm 0,45^{\mathrm{b}}$ & $5,92 \pm 0,25^{\mathrm{b}}$ \\
Carboidratos & $61,71 \pm 0,27^{\mathrm{a}}$ & $61,88 \pm 0,48^{\mathrm{a}}$ & $61,59 \pm 0,57^{\mathrm{a}}$ \\
Lipídios & $27,31 \pm 0,21^{\mathrm{a}}$ & $28,73 \pm 0,10^{\mathrm{b}}$ & $28,11 \pm 0,46^{\mathrm{b}}$
\end{tabular}

Letras iguais na mesma linha indicam que não houve diferença estatística ao nível de 5\% de significância. $\mathrm{DP}=$ desvio-padrão.

Fonte: Autoria própria.

Os biscoitos formulados apresentaram resultados satisfatórios de acordo com a legislação vigente que preconiza que a umidade de biscoitos deve ser no máximo de 14,0\% e o teor de cinzas no máximo 3,0\% (BRASIL, 1978). Nota-se que não houve diferença significativa de umidade entre os biscoitos. A fibra solúvel presente na farinha 
da banana verde, é considerada um ingrediente funcional que retêm a água dos produtos alimentícios (AGAMA-ACEVEDO et al., 2012) e a baixa umidade dos produtos pode contribuir para uma maior vida de prateleira, uma vez que torna o alimento menos propício ao desenvolvimento microbiano (FRANCO \& LANDGRAF, 2008).

Em relação ao teor de cinzas, o biscoito produzido com FBV apresentou valores superiores em relação à formulação padrão. Esse fato está relacionado a quantidade de minerais presentes na banana verde (FASOLIN et al., 2007).

Quanto aos teores de proteína avaliados, notou-se que o biscoito FP apresentou quantidade superior de proteína em comparação aos de FBV. Esse fato pode ocorrer devido a utilização de farinha de trigo, onde está presente o glúten, constituído por proteínas solúveis (albumina e globulina) e proteínas de reserva (gliadina e glutenina), e essa composição pode influenciar na quantidade de proteína encontrada na preparação (HOSENEY, 1991). O mesmo ocorreu em relação ao resultado de lipídios, visto que a farinha de trigo utilizada continha, aproximadamente, $2 \%$ desse componente, segundo a informação nutricional do rótulo, o que pode ter contribuído para a pequena diferença da formulação padrão e dos biscoitos experimentais.

Participaram do estudo de avaliação sensorial 134 provadores não treinados, sendo a maioria do sexo feminino (78\%) e a idade média foi de 18 a 25 anos (80\%), universitários (74\%), com renda média de 1 a 5 salários mínimos por mês (67\%). Todos os participantes relataram o hábito de consumir biscoitos.

Na tabela 2, pode-se observar as notas médias para os atributos cor, sabor, aroma e textura dos biscoitos desenvolvidos no presente trabalho. Para os parâmetros cor, sabor, textura e aroma, as médias encontradas equivaleram aos termos "gostei moderadamente" a "gostei muito", segundo a escala hedônica utilizada.

Tabela 2 - Médias das notas atribuídas pelos provadores dos atributos sensoriais dos biscoitos padrão (FP) e biscoitos experimentais (FE1 e FE2).

\begin{tabular}{cccc}
\hline Atributos & $\begin{array}{c}\text { FP } \\
\text { Média }+ \text { DP }\end{array}$ & $\begin{array}{c}\text { FE1 } \\
\text { Média } \pm \text { DP }\end{array}$ & $\begin{array}{c}\text { FE2 } \\
\text { Média } \pm \text { DP }\end{array}$ \\
\hline Cor & $8,09 \pm 1,33^{\mathrm{a}}$ & $7,03 \pm 1,70^{\mathrm{b}}$ & $6,84 \pm 1,65^{\mathrm{b}}$ \\
Sabor & $7,85 \pm 1,51^{\mathrm{a}}$ & $7,76 \pm 1,70^{\mathrm{a}}$ & $7,68 \pm 1,55^{\mathrm{a}}$ \\
Aroma & $7,81 \pm 1,35^{\mathrm{a}}$ & $7,49 \pm 1,51^{\mathrm{b}}$ & $7,44 \pm 1,54^{\mathrm{b}}$ \\
Textura & $8,11 \pm 1,16^{\mathrm{a}}$ & $7,83 \pm 1,53^{\mathrm{a}}$ & $7,91 \pm 1,42^{\mathrm{a}}$
\end{tabular}

Letras iguais na mesma linha indicam que não houve diferença estatística ao nível de 5\% de significância. DP = desvio-padrão.

Fonte: Autoria própria. 
A avaliação sensorial realizada demonstrou que os biscoitos apresentaram índice de aceitabilidade acima de $70 \%$ para os diferentes atributos estudados, sendo, portanto, satisfatórios (Figura 1).

Figura 1 - Índice de aceitabilidade dos biscoitos padrão (FP) e biscoitos experimentais (FE1 e FE2), segundo os atributos cor, sabor, aroma e textura

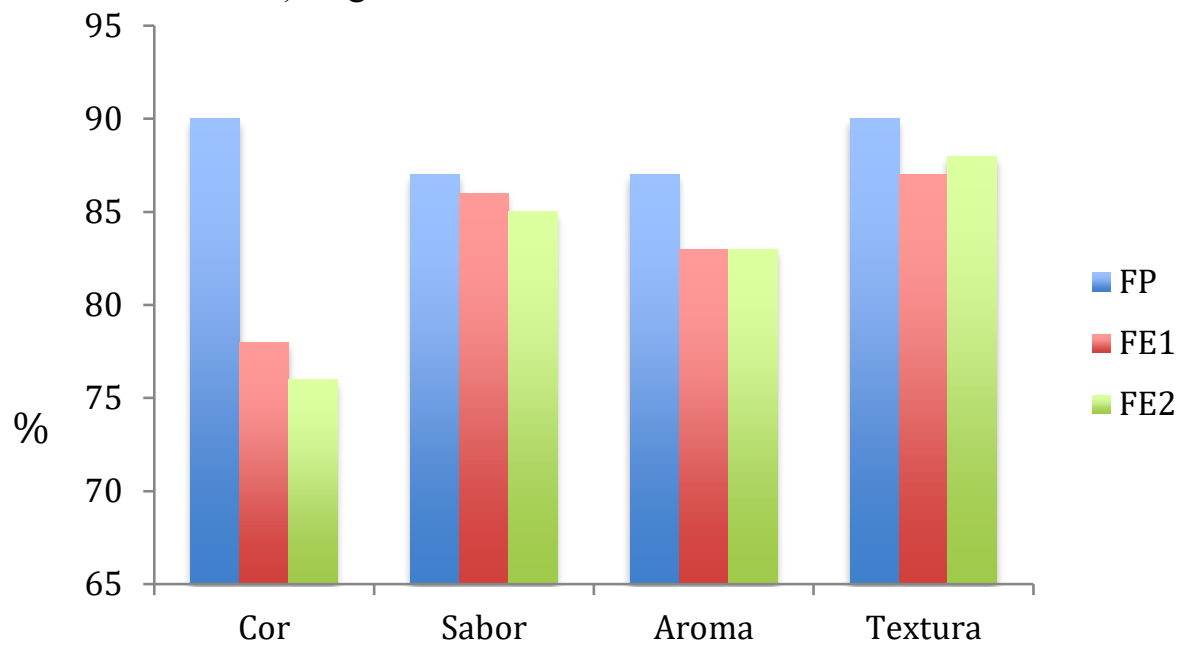

Fonte: Autoria própria.

Conforme demonstrado na Figura 2, é possível observar a frequência de intenção de compra dos produtos. A maioria dos entrevistados respondeu que compraria os biscoitos FBV ocasionalmente.

Figura 2 - Intenção de compra dos biscoitos padrão (FP) e biscoitos experimentais (FE1 e FE2).

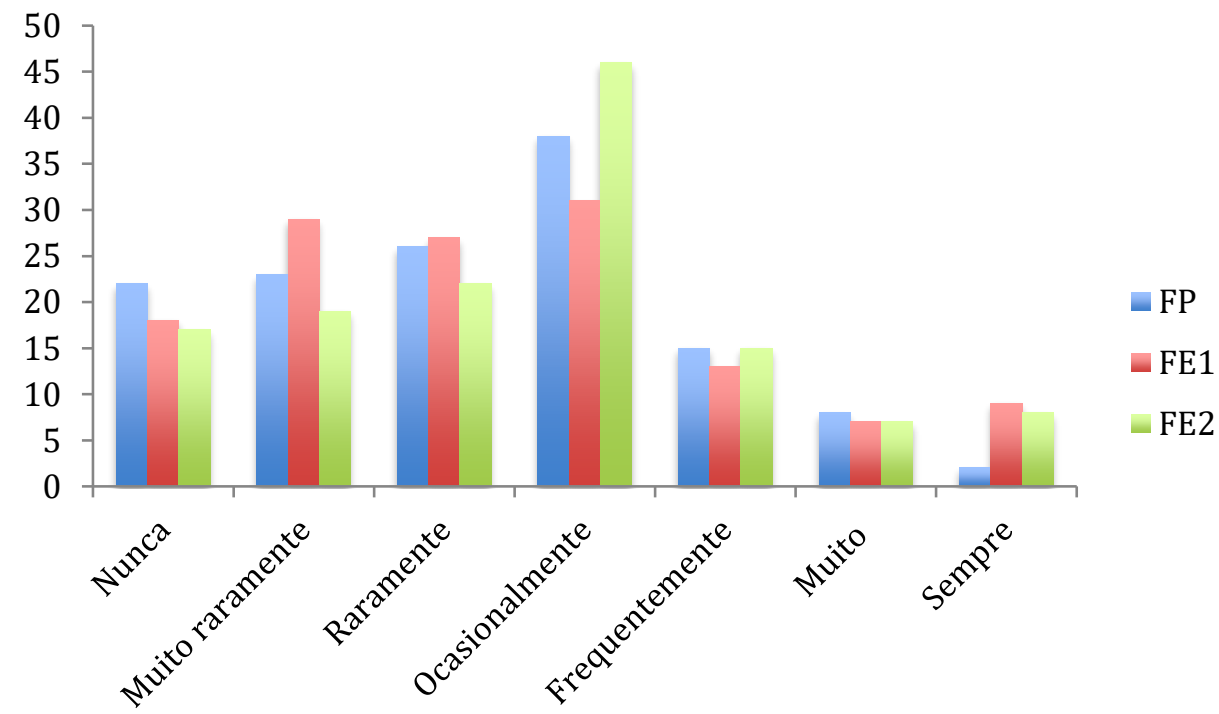

Fonte: Autoria própria. 
O uso da farinha de banana verde provocou pequenas alterações em relação à sua composição química, porém isso não parece ter prejudicado a aceitação dos biscoitos. Os biscoitos isentos de glúten elaborados com FBV podem ser uma opção para pessoas portadoras da doença celíaca, já que os celíacos relatam que a oferta de alimentos sensorialmente apropriados é restrita, o que torna a dieta monótona, e com alto custo (ARAÚJO, 2010). Além disso, a banana é plantada em todo território brasileiro, o que facilitaria a matéria-prima mais acessível (EMBRAPA, 2020).

\section{CONCLUSÃO}

Conclui-se que a farinha de banana verde, apresenta muitos benefícios ao biscoito destinado ao público celíaco, sem comprometer os atributos sensoriais e composição centesimal, possibilitando um aumento do valor nutricional do alimento e dando alternativa de um produto feito sem glúten e com aspecto sensorial atraente.

\section{REFERÊNCIAS}

AGAMA-ACEVEDO E.; HERNÁNDEZ J.J.I.; VARGAS G.P., et al. Starch digestibility and glycemic index of cookies partially substituted with unripe banana flour. Food Science and Technology, 2012; 46:77-182.

AL-BAWARDY, B; CODIPILLY, D. C.; RUBIO-TAPIA, A.; BRUINING, D. H.; HANSEL, S. L.; MURRAY, J. A. Celiac disease: a clinical review. Abdominal Radiology, Nova Iorque, Estados Unidos, v. 42, n. 2, p. 351-360, 2017.

ANDRADE, C.K.O. Elaboração e aceitabilidade dos biscoitos enriquecidos com farinha de banana verde. Monografia. Universidade Estadual da Paraíba, Paraíba; 2013.

ARAÚJO, H.M.C.; ARAÚJO, V.M.C.; BOTELHO, R. B. A.; ZANDONADI, R. P. Doença celíaca: hábitos e práticas alimentares e qualidade de vida. Revista de Nutrição, Campinas, v.23, n.3, p. 467-474, 2010.

BRASIL. Ministério da Saúde. Agência Nacional de Vigilância Sanitária. Resolução nº12 de 1978. Disponível em:

https://bvsms.saude.gov.br/bvs/saudelegis/cnnpa/1978/res0012_30_03_1978.html Acesso em: 14 dez. 2021.

DUTCOSKY, S.D. Análise sensorial de alimentos. $4^{\text {a }}$ Edição. Curitiba: Champagnat Pucpress, 2013, 531p.

EMBRAPA. Empresa Brasileira de Pesquisa Agropecuária. 2020. Disponível em: https://www.agencia.cnptia.embrapa.br/Agencia40/AG01/Abertura.html. Acesso em: $18 / 06 / 2021$. 
FASOLIN, L. H.; ALMEIDA, G. C.; CASTANHO, P. S.; NETTO-OLIVEIRA, E. R. Biscoitos produzidos com farinha de banana: avaliações química, física e sensorial. Ciência e Tecnologia de Alimentos, v. 27, n. 3, p. 524-529, 2007.

FRANCO, B.D.G. M. \& LANDGRAF, M. Microbiologia dos alimentos. São Paulo: Atheneu, 2008. 192p.

GALLAGHER, E; GORMLEY, T.R.; ARENDT, E.K. Recent advances in the formulation of gluten-free cereal-based products. Trends in Food Science $\&$ Technology, Dublin, v. 15, n. 3-4, p.143-152, 2004.

HOSENEY, R.C. Principios de Ciencia y Tecnologia de los Cereales. Zaragoza: Acribia, 1991. IX, 321p. ISBN 842000703X (Broch.).

INSTITUTO ADOLFO LUTZ. Normas analíticas do Instituto Adolfo Lutz: métodos químicos e físicos para análise de alimentos. 2.ed. São Paulo: Instituto Adolfo Lutz; 2008.

PERUCHA, V.R. Propriedades Funcionais da Banana Verde. Nutrição, Saúde e Performance - Anuário de Alimentos Funcionais, São Paulo, ed n. 26, p. 59-64, 2005.

RANIERI, L.M.; DELANI, T.C.O. Banana Verde (Musa spp): Obtenção da Biomassa e Ações Fisiológicas do Amido Resistente. Revista UNINGÁ Review, Maringá, v. 20, n. 3, p. 43-49, 2014.

RODRIGUES, Ana Sofia Medeiros. A Doença Celíaca: etiopatogenia, diagnóstico, aspetos clínicos e tratamento. 2013. 75 f. Dissertação de Mestrado - Curso de Ciências Farmacêuticas, Faculdade de Ciências da Saúde, Universidade Fernando Pessoa, Porto, 2013. 